\title{
TITLE:
}

\section{Designing Multimodal Freight Transport Networks: A Heuristic Approach and Applications}

\section{$\operatorname{AUTHOR}(\mathrm{S}):$}

Yamada, Tadashi; Russ, Bona Frazila; Castro, Jun; Taniguchi, Eiichi

\section{CITATION:}

Yamada, Tadashi ... [et al]. Designing Multimodal Freight Transport Networks: A Heuristic Approach and Applications. TRANSPORTATION SCIENCE 2009, 43(2): 129-143

\section{ISSUE DATE:}

2009-05

URL:

http://hdl.handle.net/2433/109941

RIGHT:

Copyright (C) 2009 by INFORMS. 


\title{
Designing Multimodal Freight Transport Networks: A Heuristic Approach and Applications
}

\author{
Tadashi Yamada \\ Department of Urban Management, Kyoto University, Nishikyo, Kyoto 615-8540, Japan, \\ t.yamada@kiban.kuciv.kyoto-u.ac.jp \\ Bona Frazila Russ \\ Department of Civil Engineering, Bandung Institute of Technology, Bandung 40132, Indonesia, \\ frazila@trans.si.itb.ac.id \\ Jun Castro \\ School of Urban and Regional Planning, University of the Philippines, Diliman, 1101 Quezon City, \\ Philippines, jtcastro@up.edu.ph \\ Eiichi Taniguchi \\ Department of Urban Management, Kyoto University, Nishikyo, Kyoto 615-8540, Japan, \\ taniguchi@kiban.kuciv.kyoto-u.ac.jp
}

\begin{abstract}
$\mathrm{D}$ esigning multimodal freight transport networks can facilitate the economic development of regions and countries as well as help to reduce negative environmental impacts. It is therefore crucial that such be undertaken in areas where more priority is given on road-based freight transport systems. This paper proposes a model for strategic transport planning, particularly in freight terminal development and interregional freight transport network design. The model determines a suitable set of actions from a number of possible actions, such as improving the existing infrastructure or establishing new roads, railways, sea links, and freight terminals. Modelling is undertaken within the framework of bilevel programming, where a multimodal multiclass user traffic assignment technique is incorporated within the lower-level problem, whilst the upper-level problem determines the best combination of actions such that the freight-related benefit-cost ratio is maximised. The upper-level problem involves combinatorial optimisation, and a heuristic approach based on genetic local search is applied as a solution technique. Empirical results of the model as applied to an actual large-sized interregional intermodal freight transport network show that genetic local search could provide better performance as compared to other genetic algorithm-based, as well as tabu search-based, heuristics. The model is successfully applied to transport network planning in the Philippines, where the development of a freight transport network is necessary to increase the utilisation of other modes rather than road-based vehicles.
\end{abstract}

Key words: multimodal freight transport; discrete network design; genetic local search

History: Received: February 2007; revisions received: October 2007, April 2008; accepted: October 2008.

Published online in Articles in Advance May 7, 2009.

\section{Introduction}

Freight transport constitutes an important activity undertaken between cities as well as within cities. Road-based freight transport has significantly increased, and consequently, the use and overuse of road networks have generated various externalities such as traffic congestion, increased energy consumption, and negative environmental impacts. An efficient spatial organisation of multimodal transport systems has the potential to alleviate such externalities and establish sustainable cities, regions, and countries (e.g., Rothengatter 1991, Priemus 1999, Taniguchi et al. 2001). Multimodal freight transport networks could likewise support the economic development of cities, regions, and countries, and help reduce negative impacts on the environment and energy consumption.

Multimodal transport systems are also useful in expanding the freight transport network in developing countries, where much of the focus is centerd on roadbased freight transport systems. At present, the existing multimodal facilities consisting of roads, rail, and ports in developing countries are still undeveloped. Road capacities, especially outside urban areas, are still inadequate, and several road segments have very poor conditions. In addition, most of the port terminals provide very low levels of service because of lack of berths and supporting equipment. Hence, transport infrastructures have not been optimally developed to suit the needs for a well-coordinated and efficient multimodal operation.

This paper deals with the problem of investment planning in developing multimodal freight transport networks, which can be translated to a problem of identifying and selecting a suitable set of actions from a number of possible actions. These actions include improving existing infrastructure or establishing new 
roads, railways, sea links, and freight terminals. The most feasible set of infrastructure projects is selected for efficient design of the multimodal freight transport network. The actions are represented by adding new links or selecting existing links for improvement in the transport network. The problem can therefore be considered as a discrete network design problem (DNDP), which basically implies the selection of link additions to an existing transport network with given demand from each origin to each destination. The DNDP is described in detail in Bruynooghe (1972); Steenbrink (1974); Poorzahedy and Turnquist (1982); Boyce (1984); Magnanti and Wong (1984); Chen and Alfa (1991); Yang and Bell (1998); and Gao, Wu, and Sun (2005).

As indicated in Ravi and Sinha (2006), there has been very little effort to study the problem in an integrated manner that would allow one to exploit the savings that may result from making both decisions in a coordinated way to reduce the total cost of location and transportation. In this context, Arnold, Peeters, and Thomas (2004) deals with the problem of optimally locating rail/road terminals for freight transport and relates closely to our problem. Their approach is based on fixed-charge network design problems (e.g., Magnanti and Wong 1984; Balakrishnan, Magnanti, and Wong 1989), which are also applied by Melkote and Daskin (2001) for simultaneously optimising facility locations and the design of the underlying transport network. As such, network location theory, including the fixed-charge network design problems, location-routing problems (e.g., Laporte 1988; ReVelle and Laporte 1996; Min, Jayaraman, and Srivastava 1998), and hub location problems (e.g., O'Kelly 1987; Aykin 1990; Campbell 1994, 1996) has been a powerful tool for transport network design and facility locations. Francis, McGinnis, and White (1992), Daskin (1995), and Drezner (1995) provide a comprehensive overview of network location models.

Many useful findings have been provided by these conventional network location models in efficiently obtaining exact/approximate optimal solutions and clarifying the basic relationships between the optimal location and transport cost. However, such models have not appropriately taken into account the change in traffic flow caused by expanding and improving the transport network. Traffic conditions on the transport network should be influenced by the network improvement actions implemented, especially when such actions are carried out on a larger scale as transport initiatives in interregional or urban transport planning.

The model presented in this paper explicitly incorporates traffic and freight flows on the transport network using a multimodal multiclass user equilibrium traffic assignment technique (e.g., Dafermos 1980; Crainic, Florian, and Leal 1990; Guelat, Florian, and Crainic 1990; Cascetta 2001; Nagurney and Dong 2001). This allows the model to deal with more realistic situations. Therefore, the model has the potential to be used as a tool for strategic level of multimodal freight transport planning, particularly in freight terminal development and freight transport network design. To our knowledge, there has been no research that uses such traffic assignment techniques in DNDP, with the performance of the model being investigated with actual large-sized interregional intermodal freight transport networks. The application of the model to such transport networks is the highlight of the paper.

The optimisation process in determining the set of network improvement actions is described within the framework of bilevel programming, where the multimodal multiclass user equilibrium traffic assignment technique is incorporated within the lower level, whilst the combination of actions is approximately optimised using metaheuristics-based procedures in the upper level. This type of problem also involves a mathematical problem with equilibrium constraints (MPEC) (Luo, Pang, and Ralph 1996). The advantage of adopting metaheuristic techniques is that such techniques can handle complex problems and provide the flexibility of the design of bilevel programming problems if applied as optimisation techniques. These techniques can also compute approximately efficient solutions in relatively shorter times. Therefore, there have been an increasing number of researches incorporating metaheuristic techniques into such problems (e.g., Bard 1998, Taniguchi et al. 1999, Shepherd and Sumalee 2004, Zhang and Yang 2004).

This sort of bilevel programming is similar to that used in Taniguchi et al. (1999). However, the model presented in this paper can handle both the improvement of link capacity and the addition of new links to an existing multimodal transport network and can deal with a variety of actions relating to the development of freight transport network, whereas the model described in Taniguchi et al. (1999) can only deal with the choice of nodes for pickup/delivery and line-haul trucks to determine the location and size of freight terminals taking the behaviour patterns of motorists into consideration. This difference depends partly on the use of multimodal multiclass user equilibrium traffic assignment technique to be formulated with variational inequality, which is not applied in Taniguchi et al. (1999). Furthermore, this paper utilises more detailed representation of multimodal transport networks and deliberates on the choice of metaheuristic techniques to be incorporated within the model.

The rest of the paper is organised as follows. In the following section, the overall modelling framework is 
described, and the formulation of the model is given. In §3, the performance of several heuristic procedures to be incorporated within the upper level is investigated on a test network with the objective of applying the model to an actual large-sized interregional intermodal freight transport network. The model is then tested and applied in $\S 4$ to an actual transport network in the Philippines, and explores the implications of how to expand the interregional freight transport network. Finally, in $\S 5$, the methodologies, results, and analyses in the paper are summarised.

\section{The Model}

Modelling is undertaken using bilevel programming. The lower level describes the multimodal multiuser equilibrium flow on the transport network, whereas the upper level determines the best combination of network improvement actions. Overall, the model can be represented as a leader-follower game (Stackelberg 1934), where transport planning departments are the leaders, and the followers are the transport network users (i.e., freight and passengers). This model can simply be formulated as follows (where $\langle\cdot, \cdot\rangle$ denotes the inner product):

$$
\begin{array}{rl}
\max _{\mathbf{y}} & f\left(\mathbf{y}, \mathbf{b}, \mathbf{x}^{*}, \mathbf{x}_{0}^{*}\right) \\
\text { s.t. } & \left\langle\mathbf{c}\left(\mathbf{x}^{*}\right), \mathbf{x}-\mathbf{x}^{*}\right\rangle \geq 0 \quad \forall \mathbf{x} \in \mathrm{K}, \\
& \left\langle\mathbf{c}\left(\mathbf{x}_{0}^{*}\right), \mathbf{x}_{0}-\mathbf{x}_{0}^{*}\right\rangle \geq 0 \quad \forall \mathbf{x}_{0} \in \mathrm{K}_{0},
\end{array}
$$

where,

y: set of freight network improvement actions,

b: vector of investment/operation costs,

$\mathbf{x}$ : vector of link flows,

$\mathbf{x}^{*}$ : vector of equilibrium link flows,

$\mathbf{x}_{0}$ : vector of link flows without any action implemented,

$\mathbf{x}_{0}^{*}$ : vector of equilibrium link flows without any action implemented,

c: vector of link user costs,

$K, K_{0}$ : feasible constraint set.

The upper-level problem uses the benefit-cost ratio (BCR) as an objective function in this paper to identify the economic effectiveness of freight network improvement actions. The benefits gained from the set of actions implemented are strongly influenced by traffic conditions on the transport network, and thus the flows of freight and passengers on it are described within the lower-level problem (i.e., Constraints (2) and (3)) using a user equilibrium traffic assignment technique.

\subsection{Lower-Level Problem}

The lower-level problem utilises a modal splitassignment model. This is an aggregate-type multimodal freight transport network model on the basis of strategic freight network planning models (e.g., Friesz,
Tobin, and Harker 1983; Crainic, Florian, and Leal 1990; Guelat, Florian, and Crainic 1990; Tavasszy 1996; Southworth and Peterson 2000). The reason why such an aggregate approach was used is that the national origin-destination and transport facility surveys in the Philippines are almost viable for interregional freight transport modelling. These only offer aggregate origin-destination information that identifies the mode shares of the total commodity but cannot provide information on the activities of individual shippers as well as those of carriers. Thus, neither the influence of shipper-carrier behaviour nor their interaction in the freight transport decision is explicitly taken into account in this model.

The model allows freight and passengers to be treated as multiclass users, with modal split and route choice carried out simultaneously by converting the multimodal network into a unimodal abstract mode network. A detailed representation of freight movement within terminals is developed based on network descriptions proposed by Guelat, Florian, and Crainic (1990); Tavasszy (1996); and Southworth and Peterson (2000), incorporating loading/unloading, storage and administrative processes (see Figure 1).

The lower-level problem involves user equilibrium conditions with a nonseparable and asymmetric Jacobian matrix cost function among user types that can be stated using variational inequality (e.g., Dafermos 1980, Nagurney 2000). This can be formulated as follows:

Find $x_{a}^{i^{*}} \in \mathbf{\kappa}$ such that:

$$
\sum_{i=1}^{p} \sum_{a \in A} c_{a}^{i}\left(\tilde{\mathbf{x}}^{*}\right) \times\left(x_{a}^{i}-x_{a}^{i^{*}}\right) \geq 0 \quad \forall \tilde{\mathbf{x}} \in \mathbf{\kappa},
$$

where $x_{a}^{i^{*}}$ is the user equilibrium flow of link $a$ for user type $i$, and $\tilde{\mathbf{x}}$ is a $p$-dimensional column vector with the components $\left\{x_{a}^{1}, \ldots, x_{a}^{p}\right\} . \mathbf{\kappa}$ is defined as $\boldsymbol{\kappa} \equiv\{\tilde{\mathbf{x}} \mid$ satisfying the nonnegative path flows and conservation of flow $\}. c_{a}^{i}($.$) is the generalised cost on link a$ for

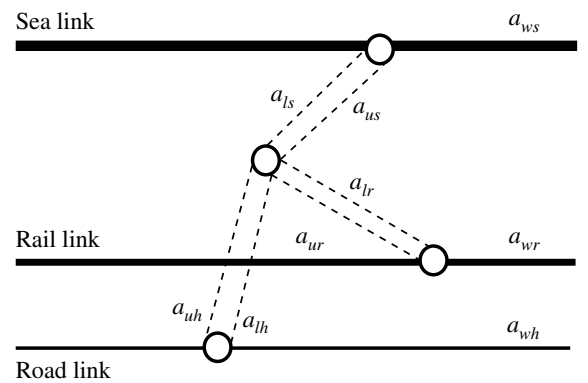

Figure 1 Multimodal Transport Network Representation

Notes. $\mathrm{a}_{s}$ : loading link for sea mode; $\mathrm{a}_{u s}$ : unloading link for sea mode; $\mathrm{a}_{l r}$ : loading link for rail mode; $\mathrm{a}_{u r}$ : unloading link for rail mode; $\mathrm{a}_{l h}$ : loading link for road mode; $a_{u h}$ : unloading link for road mode; $a_{w s}$ : link used by sea mode (including waiting for vehicle); $\mathrm{a}_{w r}$ : link used by rail mode (including waiting for vehicle); $a_{w h}$ : link used by road mode; $\mathbf{O}$ node. 
user type $i$, and $A$ is the set of links on the transport network. Hence, the model incorporates the diagonalisation method (e.g., Florian and Spiess 1982, Sheffi 1985, Thomas 1991) within its solution procedures.

Generalised link costs are described to be made up of a fare component and a time cost component (see Equation (6)). The time spent on the link includes travel time, waiting time, and loading/unloading time, depending on the type of links. Link types are classified into link ways and terminal links for each mode (see Figure 1). Although the fare component is assumed to have a fixed value and does not depend on volume, the time cost component, particularly the time spent on the link, is a function of volume and differs by link type. The time spent on the link is represented by a continuous function in the form of polynomial approximation presented by Crainic, Florian, and Leal (1990) to keep the link cost function monotonically increasing (see Equation (7)).

\subsection{Upper-Level Problem}

The upper-level problem approximately optimises the combination of freight network improvement actions based on the ratio of the reduced total generalised freight cost and the investment/operational cost incurred for implementing the actions. This can be undertaken using the benefit-cost ratio. Therefore, the objective function for the upper-level problem is to maximise the $B C R$ value of a combination of actions to be implemented.

Let $A^{\prime}$ be defined as $A^{\prime}=A_{1} \cup A_{2} \cup A_{3}$, where $A_{1}=$ $\{a: a=1,2, \ldots, n\}$ is the set of existing links without modifications, $A_{2}=\{a: a=n+1, n+2, \ldots, n+m\}$ is the set of existing links with possible actions to be implemented, and $A_{3}=\{a: a=n+m+1, \ldots, n+2 m\}$ is the updated set of $A_{2}$ after the associated action is implemented. Links in $A_{2}$ and $A_{3}$ can be numbered such that if $a \in A_{2}$ is selected (i.e., if the action associated with it is implemented), link $a+m$ in $A_{3}$ will replace $a$; otherwise, $a+m$ in $A_{3}$ will be discarded.

Here, let $\mathbf{y}$ be defined as the set of possible actions associated with $A_{3}$ (or $A_{2}$ ), $\mathbf{y}=\left\{y_{a} \mid a=n+m+1\right.$, $n+2 m\}\left(\right.$ or $\left.\mathbf{y}=\left\{\mathrm{y}_{\mathrm{a}} \mid a=n+1, n+m\right\}\right)$, where $y_{a}$ is the action implementation indicator with a binary value of 1 if the action related to link $a$ on the set of $A_{2}$ is implemented, and 0 if it is otherwise, the objective function, $f(\mathbf{y})$, can be represented as follows:

$$
\begin{aligned}
& \max f(\mathbf{y}) \\
& =\frac{\sum_{i \in F}\left(\sum_{a \in A_{1} \cup A_{2}} x_{0 a}^{i^{*}} c_{a}^{i}\left(x_{0 a}^{i^{*}}\right)-\sum_{a \in A_{1}} x_{a}^{i^{*}} c_{a}^{i}\left(x_{a}^{i^{*}}\right)-\sum_{a \in A_{2}} x_{a}^{i^{*}} c_{a}^{i}\left(x_{a}^{i^{*}}, y_{a}\right)\right)}{\sum_{a \in A_{2}} b_{a} y_{a}},
\end{aligned}
$$

where,

$$
\begin{gathered}
c_{a}\left(x_{a}^{i}, y_{a}\right)=\rho_{a}^{i}+\alpha^{i} d_{a}^{i}\left(x_{a}^{i}, y_{a}\right), \\
d_{a}^{i}\left(x_{a}^{i}, y_{a}\right)=t_{a}\left[1+\phi_{1} x_{a}^{i}+\phi_{2}\left(\frac{x_{a}^{i}}{r_{0 a}+y_{a} r_{a}}\right)^{\gamma}\right],
\end{gathered}
$$

where,

$F$ : set of user types for freight transport,

$x_{a}^{i}$ : flow on link $a$ for user type $i$,

$x_{0 a}^{i^{*}}$ : flow on link $a$ for user type $i$, which is the solution to the lower-level problem without any action implemented,

$b_{a}$ : investment/operation cost for link $a$,

$\rho_{a}^{i}$ : fare on link $a$ for user type $i$,

$\alpha^{i}$ : time value for user type $i$,

$d_{a}^{i}\left(x_{a}^{i}\right)$ : time spent on link $a$ for user type $i$,

$t_{a}$ : free travel time on link $a$,

$r_{0 a}$ : existing link capacity on link $a$,

$r_{a}$ : additional link capacity on link $a$ if the action is implemented,

$\phi_{1}, \phi_{2}, \gamma$ : parameters to be calibrated.

The objective function shown in Equation (5) represents the BCR value, which is the ratio of reduced total generalised freight cost and investment/ operation cost required for implementing the set of actions. The reduced total generalised freight cost is considered as the benefit produced by the implemented set of actions and can be computed as the difference between the total generalised cost incurred with and without the actions implemented. Combining the actions can generally yield synergistic effects depending on the actions included. The generalised cost incurred on each link can be estimated by Equation (6), which is composed of a fare component and a time cost component, with the time spent on each link being assessed by Equation (7). Budget constraints on the investment/operation cost are not taken into account in this paper, even though this could be possible by incorporating additional procedures within the solution techniques for the upper-level problem.

$B C R$ values are commonly estimated using the total benefits and costs generated for the expected duration of the action to be implemented. However, in the subsequent testing and application of the model, because the future OD flows for both freight and passengers have hardly been estimated accurately yet, the equivalent one-year benefit and cost are used. In this context, the analyses presented in this paper are only preliminary, and more detailed BCR analysis should be conducted in the future, taking into account the project life and social discount rates.

The upper level can be considered as a combinatorial optimisation problem. Metaheuristics-based procedures are used to solve the problem. In the past decade, several metaheuristics have been developed and applied in the field of soft computing. A vital role of these techniques is to solve complex and difficult mathematical programming problems, which often involve NP-hard problems. These cannot ensure obtaining exact optimal solutions, but can provide reasonable and practical solutions. Hence, 
these have been commonly applied to combinatorial optimisation problems wherein exact optimal solutions are hard to determine. Ribeiro and Hansen (2001), Michalewicz and Fogel (2002), Glover and Kochenberger (2003), Herz and Widmer (2003), and Resende and Pinho de Sousa (2004) provided excellent introductions and basic concepts of metaheuristics. In general, genetic algorithms (GA) (e.g., Holland 1975, Goldberg 1989, Davis 1991, Reeves 1997), tabu search (e.g., Glover and McMillan 1986, Glover and Laguna 1997), simulated annealing (e.g., Kirkpatrick, Gellat, and Vecchi 1983; Aarts and Korst 1989), and ant colony optimisation (e.g., Dorigo, Di Caro, and Gambardella 1999; Dorigo and Stutzle 2004) are typical solution techniques in metaheuristics.

This paper adopts the genetic local search (GLS) method (e.g., Ackley 1987, Radcliffe and Surry 1994, Jaszkiewicz 2002, Jaszkiewicz and Kominek 2003, Arroyo and Armentano 2005), which is a hybrid metaheuristic technique combining genetic algorithms with local search. GLS, sometimes called memetic algorithms or hybrid genetic algorithms, also involves an evolutionary computation technique on the basis of GA. Heuristics based on the GLS scheme often outperform other metaheuristics on combinatorial optimisation problems (e.g., Murata and Ishibuchi 1994, Merz and Freisleben 1997, Galinier and Hao 1999), and this paper is motivated by such successful implementations of GLS-based heuristics for combinatorial optimisation problems. A possible explanation on the efficiency of GLS is given by Jaszkiewicz (2002), that in many cases, local optima constitute a relatively small part of the search space and thus can be achieved in an efficient way. GLS offers the potential to cover the weakness of GA in searching local areas because GA can efficiently find the vicinity of the optimal solution from a wider range.

The details of GLS applied in this paper are described as follows:

\section{Parameters:}

size of the current population: $N$

stopping criterion: predefined number of generations (i.e., number of iterations given in advance)

Initialization:

Current population $\mathbf{y}:=\varnothing$

Current generation $l=0$

repeat $N$ times

Generate a feasible solution (i.e., an individual) $y_{a}$ by a randomized algorithm

Add $y_{a}$ to $\mathbf{y}$ for constructing an initial population

Main loop:

repeat

Select $K$ best individuals to make $\mathbf{y}_{1}$ (i.e., Elitist selection)
Evaluate $\mathbf{y}$ and select $(N-K)$ individuals according to their fitness to obtain $\mathbf{y}_{2}$

Draw two solutions $(N-K)$ times from $\mathbf{y}_{2}$ as parent solutions

Generate $(N-K)$ child solutions (i.e., offspring) to obtain $\mathbf{y}_{3}$ by applying uniform crossover procedure with predefined crossover rate

Mutate each bit of $(N-K)$ child solutions in $\mathbf{y}_{3}$ with some low probability to obtain $\mathbf{y}_{4}$

Apply local search to $\mathbf{y}_{4}$ to obtain $\mathbf{y}_{5}$

Develop current population $\mathbf{y}$ consisting of $\mathbf{y}_{1}$ and $\mathbf{y}_{5}$

Change $l$ to $l+1$

until the stopping criterion is met

\section{Empirical Comparison Study}

\subsection{GA-Based Procedures}

Three types of GA-based procedures are tested to compare their performance for identifying the superiority of GLS. In the initial generation (i.e., in the case of $l=0$ in the algorithm described above) for all the three procedures, a specific number of sets of actions (i.e., specific number of individuals) is generated and its chromosomes are randomly created. The chromosomes are formed in such a way that the action implementation indicator takes a binary value of 1 if the action is implemented and 0 if it is otherwise. For example, for a feasible solution containing the four actions of 1, 2, 15, and 16 to be implemented among a total of 16 possible actions, the corresponding individual is represented as $[1,1,0,0,0,0,0,0,0,0$, $0,0,0,0,1,1]$. The value of the objective function given by Equation (5) is then calculated for each individual, and its fitness is evaluated. Succeeding operators vary accordingly among the three procedures. The outline of the three schemes is described below.

(i) Simple Genetic Algorithm (SGA)

SGA is the basic version of the GA-based scheme with standard operators in selection and reproduction, crossover, and mutation (e.g., Goldberg 1989, Davidor 1991). Here, linear fitness scaling (Davis 1991) is used in the selection and reproduction process. Single-point crossover is applied with its location and the pairs of parents being randomly selected, and creep mutation is adopted.

(ii) GA improved with additive operators (GA-AO)

Elitist selection (e.g., Goldberg 1989) is used to preserve some of the best individuals for further generation. Mutation process is the same as that adopted in SGA, but uniform crossover (e.g., Syswerda 1989) is applied in the crossover process, where the location of the crossover and the pairs of parents are also selected at random. Taniguchi et al. (1999) and Yamada, Taniguchi, and Noritake (1999) identify that 


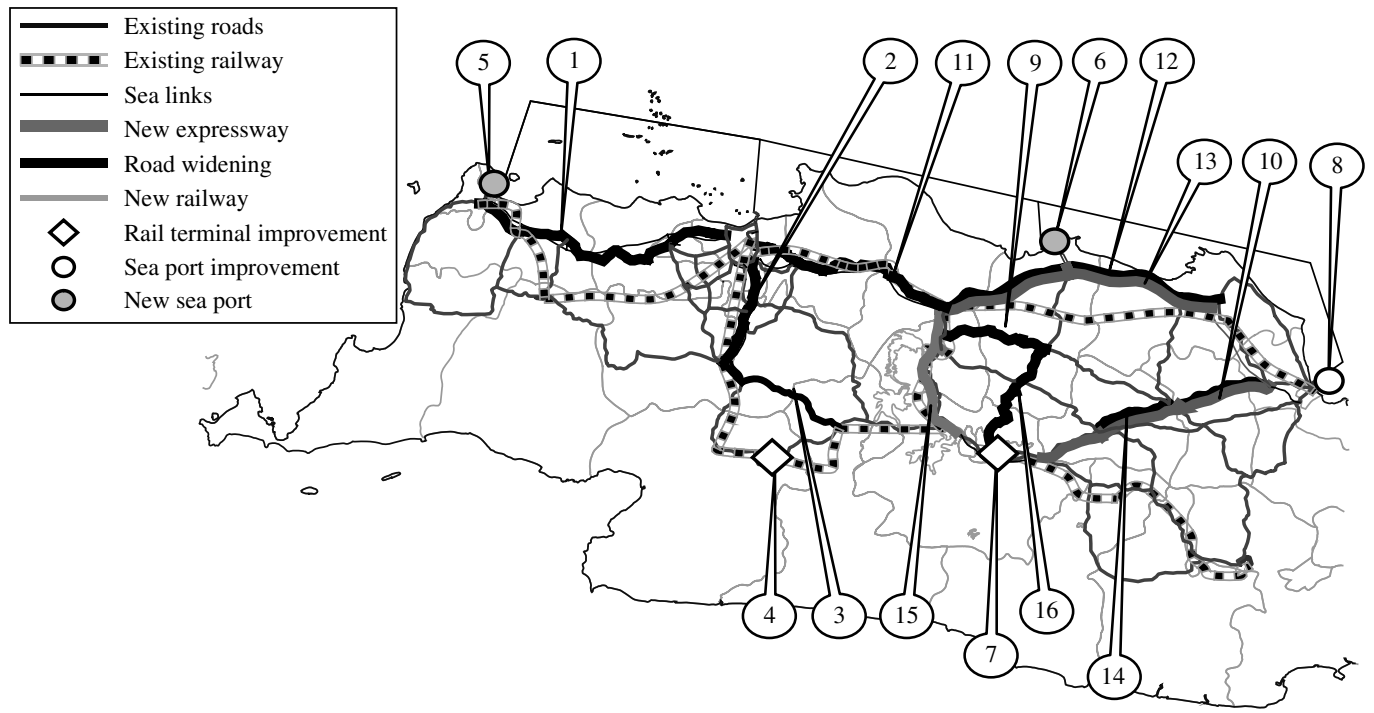

Figure 2 Test Network and Actions (West Java)

these procedures can provide better performance in combinatorial optimisation problems.

(iii) Genetic Local Search (GLS)

GLS is a procedure that integrates local search techniques with GA. As shown in the previous section, a local search operator is inserted after the mutation process of the GA-AO procedure. Here, a local search operator for swapping the neighbourhood is utilised, where a gene is randomly selected in every individual, and each of the neighbouring genes is swapped for its allele. This can produce three individuals, including the original one. The best individual among these three is determined and preserved for the next generation. This can be considered as a kind of "best admissible move strategy" (e.g., Yagiura and Ibaraki 2001).

Parameter values for these operators are determined based on the findings of existing research by Goldberg (1989), Taniguchi et al. (1999), and Yamada, Taniguchi, and Noritake (1999). The length of the chromosome is assumed to be 16 (i.e., $m=16$ ), which is the number of actions used in the succeeding model application to the transport network in the Philippines. The crossover rate is set to 0.6 and the mutation rate to 0.03 . The number of elites preserved in each generation is 10 (i.e., $K=10$ ), whereas 50 individuals (i.e., $N=50$ ) are set in each generation. The number of generations (i.e., stopping criterion) is set to 100. Existing research indicates that these parameter values are reliable for SGA and GA-AO. However, GLS might have more appropriate values for crossover and mutation rates. Therefore, the same crossover and mutation rate values as those used in existing research (i.e., crossover rate is 0.6 and mutation rate 0.03 ) are applied to investigate the effectiveness of incorporating the local search operator within the GA-AO procedure, and then the best crossover and mutation rates in GLS are explored.

The model is initially tested on a relatively small transport network in West Java, Indonesia (see Figure 2) to investigate the performance of the three types of GA-based procedures. This network is composed of 422 links, 105 nodes, 46 OD pairs for freight, and 52 OD pairs for passenger. There are 16 alternative actions, as shown in Figure 2 and Table 1, including planned and hypothesised infrastructure projects. Improvements, including road widening, are set on the assumption that the overall capacity is improved 1.5 times the initial capacity. In the case of seaport and rail terminal improvement, increased capacities

Table 1 Listing of Actions (West Java Case)

\begin{tabular}{ccccc}
\hline No. & Type of action & Location & $\begin{array}{c}\text { Investment/operation } \\
\text { cost (bil. Rp.) }\end{array}$ & BCR* \\
\hline 1 & Road widening & Jakarta-Merak & $1,474.81$ & 0.20 \\
2 & Road widening & Jakarta-Bogor & 611.58 & 0.52 \\
3 & Road widening & Padalarang-Bogor & $1,907.93$ & 0.02 \\
4 & Rail terminal & Sukabumi & $5,012.97$ & 0.02 \\
& improvement & & & \\
5 & New seaport & Ciwandan & $15,038.90$ & 0.18 \\
6 & New seaport & Karawang & $15,038.90$ & 0.04 \\
7 & Rail terminal & Bandung & $4,010.37$ & 0.16 \\
& improvement & & & \\
8 & Seaport & Cirebon & $10,025.93$ & 0.0001 \\
& improvement & & & \\
9 & Road widening & Subang-Wado & $1,156.99$ & 0.20 \\
10 & New expressway & Bandung-Cirebon & $3,958.24$ & 0.02 \\
11 & Road widening & Karawang-Jakarta & $1,769.58$ & 0.56 \\
12 & Road widening & Cirebon-Karawang & $1,705.41$ & 0.003 \\
13 & New expressway & Cikampek-Cirebon & $7,855.32$ & 0.04 \\
14 & Road widening & Sumedang-Cirebon & $1,670.32$ & 0.13 \\
15 & New expressway & Cikampek-Padalarang & $2,536.56$ & 0.36 \\
16 & Road widening & Bandung-Subang & 751.94 & 0.0003 \\
\hline
\end{tabular}

${ }^{*} \mathrm{BCR}$ of each action represents the value in case it is individually implemented. 
for loading and unloading links are assumed. Overall, the development of new expressways and seaports is relatively more expensive, whereas road widening is relatively cheaper compared with the other types.

Data obtained from the 2001 National OD Survey of Indonesia are used as inputs for interregional freight and passenger movement. Parameter values relating to link performance are mainly estimated using statistical data on roads, rails, ports, and terminals collected from various sources.

Road capacities and speed data are acquired from the database of the Indonesian Inter-urban Road Management System (IRMS) and the Indonesian toll road operator PT Jasa Marga. Railway data and related information are obtained from the Department of Communications and the semiprivate railway company PT KAI. Port information and other sea network data are collected from the Directorate General of Sea Communication under the Department of Communications. Capacities for loading/unloading links are derived from the ship-handling capacities of various ports and terminals. Time components for inspection, inventory, and administration of terminals are assumed, ranging from 6 to 48 hours depending on terminal type. The number of berths in ports is derived from the port's berth length and the average ship length for loading/unloading at sea terminals, whereas for rail terminals it is equal to the number of yards.

The best solution is found to contain action numbers 2 and 9 . The test network is relatively small, just a part of Java Island, and road-based freight transport has mainly been undertaken in this area. Hence, road widening could be more effective to improve freight transport in this case. These actions could result in the alleviation of severe traffic congestion around Jakarta, the capital of Indonesia, because of implementation of action number 2 , as well as contributing to efficient transport movements between the eastern and western parts of the region because of action number 9 .

The performance of all three procedures are compared in Table 2 and Figures 3 and 4. The results shown in Table 2 are obtained after each procedure is tested for a total of 20 runs with different seeds of random values, because all three procedures incorporate several randomised processes that affect their

Table 2 Computational Results for GA-Based Procedures (20 Runs)

\begin{tabular}{lccc}
\hline & GLS & GA-A0 & SGA \\
\hline Best & 1.45 & 1.45 & 1.45 \\
Average & 1.45 & 1.30 & 1.13 \\
Worst & 1.45 & 0.68 & 0.18 \\
Ave. CPU time (sec) & 896 & 832 & 568 \\
\hline
\end{tabular}

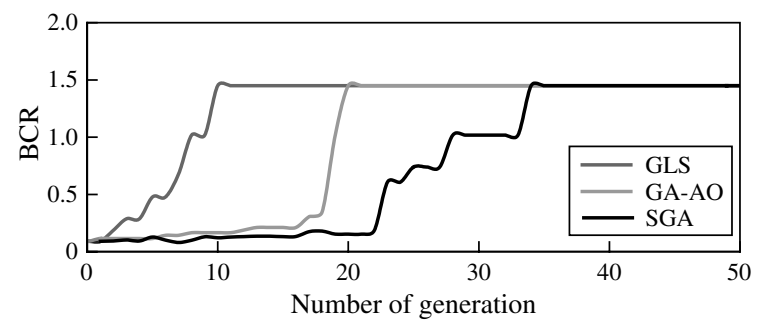

Figure 3 Highest BCR Value in Each Generation

computational results. In contrast, both Figures 3 and 4 indicate typical results for 20 computations. Table 2 reports the BCR values for the best, average, and worst solutions found across the 20 runs, as well as the average computational times in seconds to complete the search.

GLS provides consistently better performance in terms of the best, average, and worst solutions found (Table 2). Although both GA-AO and SGA have the potential to produce the same best value as GLS, their performance is not stable. In addition, it can be seen from Figure 3 that although GLS has already found the best solution in the earlier generation, SGA has not yet reached the best solution even in the 30th generation.

The average value of the objective function illustrates the convergence level of the procedures (Figure 4). GLS has nearly reached convergence after the 15 th generation, whereas other procedures are still far from convergence even in the 30th generation and do not appear to sufficiently converge even around the 100th generation.

Average computational times spent for 100 generations were measured with a PC of $3.4 \mathrm{GHz} \mathrm{CPU}$ and 1.0 GB RAM. Computational times are in part influenced by the number of individuals evaluated. GLS needs more individuals to be assessed, because it incorporates the local search algorithm. However, the accumulated number of evaluated individuals for GLS is 1,808 in the 100th generation $(1,476$ in the 30th generation), which is only $2.8 \%(2.3 \%)$ of the total number of possible combinations for the 16 alternatives.

The results indicate that GLS can provide the best performance among the three GA-based schemes and

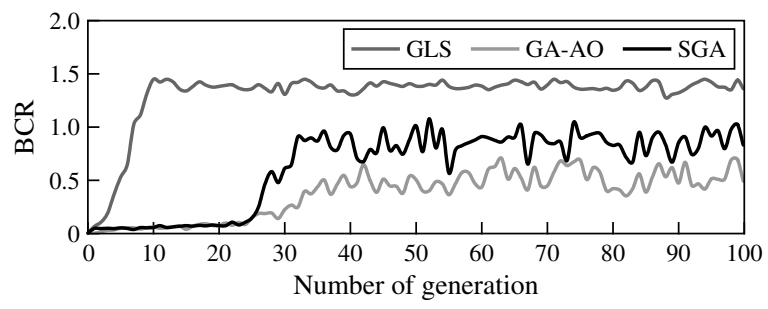

Figure 4 Average BCR Value in Each Generation 
leads to a sufficiently good solution even in the 30th generation. This implies that the local search operator embedded within the GA-AO procedure works well to supplement the performance of GA in searching local areas. When the total number of actions is 16, the number of generations (i.e., stopping criterion) in GLS can therefore be set to 30, which is the case for subsequent testing, comparisons, and applications. A lower number of generations and individuals can offer savings in computational times. This is effective particularly when the model is applied to a larger-sized transport network, because the lower-level problem requires relatively considerable computational times.

Existing GLS methods have not always encompassed both the processes of mutation and local search. Therefore, the performance of GLS without mutation should be tested to compare with that of GLS with mutation. The test is conducted with the same parameter values as those used in the experiment for the three GA-based procedures, apart from the number of generations being set to 30 . The results of the BCR values for the best, average, and worst solutions found across the 20 runs are 1.45, 1.30, and 0.45 , respectively, and the average computational time for GLS without mutation is 478 seconds (cf., the average computational time for GLS with mutation is 481 seconds for 30 generations, as shown in Table 4). These results indicate the effectiveness of incorporating mutation process within GLS in terms of robustness and superiority of solutions, as compared to the previous results of GLS with mutation shown in Table 2. This also implies a sort of synergistic effect of using both mutation and local search operators. With more genes being selected in the local search, the performance of GLS without mutation could be different from the above. However, this could lead to an increase in computational time, which could not be suitable for larger-sized freight transport networks.

The proper crossover and mutation rate values of GLS with mutation are then examined for subsequent applications of the model to actual large-sized transport networks. The test is undertaken with the same parameter values as those applied except for the crossover and mutation rates (i.e., length of chromosomes: 16, number of individuals: 50, number of generations: 30 , number of elites: 10). Table 3 compares the best, average, and worst solutions found across the 20 runs within the range of 0.4 and 0.8 for crossover rate and 0.01 and 0.05 for mutation rate. Each combination of crossover and mutation rates given in Table 3 can provide the best solution of 1.45, but the average and worst solutions vary depending on the crossover and mutation rates adopted. It can empirically be concluded that when the crossover rate is between 0.5 and 0.7 and the mutation rate is between 0.03 and 0.05 , GLS can offer relatively
Table 3 Performance Comparison in GLS (20 Runs)

\begin{tabular}{lccccc}
\hline & \multicolumn{5}{c}{ Crossover rate } \\
\cline { 2 - 6 } Mutation rate & 0.4 & 0.5 & 0.6 & 0.7 & 0.8 \\
\hline 0.01 & & & & \\
$\quad$ Best & 1.45 & 1.45 & 1.45 & 1.45 & 1.45 \\
Average & 1.20 & 1.41 & 1.36 & 1.30 & 1.31 \\
$\quad$ Worst & 0.38 & 0.68 & 0.44 & 0.68 & 0.20 \\
0.02 & & & & & \\
$\quad$ Best & 1.45 & 1.45 & 1.45 & 1.45 & 1.45 \\
Average & 1.41 & 1.41 & 1.37 & 1.41 & 1.22 \\
$\quad$ Worst & 0.68 & 0.68 & 0.68 & 0.68 & 0.68 \\
0.03 & & & & & \\
$\quad$ Best & 1.45 & 1.45 & 1.45 & 1.45 & 1.45 \\
Average & 1.41 & 1.45 & 1.45 & 1.41 & 1.37 \\
$\quad$ Worst & 0.74 & 1.45 & 1.45 & 0.68 & 0.68 \\
0.04 & & & & & \\
$\quad$ Best & 1.45 & 1.45 & 1.45 & 1.45 & 1.45 \\
Average & 1.41 & 1.45 & 1.41 & 1.45 & 1.37 \\
$\quad$ Worst & 0.68 & 1.45 & 0.68 & 1.45 & 0.68 \\
0.05 & & & & & \\
$\quad$ Best & 1.45 & 1.45 & 1.45 & 1.45 & 1.45 \\
Average & 1.45 & 1.45 & 1.45 & 1.41 & 1.37 \\
$\quad$ Worst & 1.45 & 1.45 & 1.45 & 0.68 & 0.68 \\
\hline
\end{tabular}

good and sound solutions for this type and size of problems. Higher crossover and mutation rates are, however, likely to entail more computational times because of the increased number of computational operations required for both the crossover and mutation procedures. Hence, a crossover rate of 0.5 and a mutation rate of 0.03 are hereafter used in GLS.

\subsection{GLS, TS-Based Procedures, and RSM}

Performance comparison with other metaheuristics and approximate techniques is helpful for further investigating the performance of GLS. In general, the performance of metaheuristics can often be greatly improved using adjusted parameters and procedurespecific operators. Such comparisons, therefore, might not be necessarily useful. However, Arroyo and Armentano (2005) point out that suitable implementation designs of tabu search (TS) can lead to algorithms that are very competitive to genetic algorithms in single-objective optimisation. Thus, this paper also investigates the performance of TS-based procedures as compared to that of the GLS proposed. The comparison is undertaken with random search method (RSM) apart from the TS-based procedures.

As noted in Rochat and Taillard (1995, p. 149), "A fundamental principle of TS is to exploit the interplay between diversification and intensification where diversification drives the search to examine new regions, and intensification focuses more intently on regions previously found to be good." Potentially, TS has many ways of improving its performance on specific problems, with the procedures associated with 
the diversification or with the intensification being incorporated. Glover and Laguna (1997) presents a variety of such improvements. This paper focuses on the intensification-oriented procedures rather than the diversification-oriented ones, because the GLS schemes presented above can provide better solutions searching in relatively small space (i.e., small number of individuals and generations).

The TS-based procedures used in this paper are outlined as follows:

(i) Tabu Search-Basic (TS-B)

TS-B can be defined as the fundamental version of TS. This starts with a feasible solution $\left(y_{a}\right)$ generated randomly. Repetitively, modifications of the current solution are examined by the process of move, searching for possible next solutions $\left(y_{a}^{\prime}\right)$ in the neighbourhood of the current solution (i.e., $y_{a}^{\prime} \in N\left(y_{a}\right)$ ), even if this causes a deterioration in the value of the objective function. In this paper, if the number of the neighbourhood solutions is set to 3, move is performed by randomly selecting a gene and swapping each of the neighbouring gene for its allele. This scheme is similar to the local search incorporated within GLS. The best one among the three neighbourhoods found is chosen as the current solution.

To avoid cycling, TS-B utilises the simplest form of adaptive memory, namely tabu list (i.e., tabu restrictions or short-term memory), with its tabu tenure (i.e., the size of the tabu list) being defined in advance. A simple aspiration criterion is also included within TS-B for removing the tabu status of a solution if the value of the solution is better than that of the current best solution.

(ii) Tabu Search with Multistart Method (TS-MSM)

Rochat and Taillard (1995) states that intensification typically operates by restarting from high-quality solutions. Accordingly, TS-MSM incorporates a multistart method into TS-B. After a given number of iterations, an elite solution (i.e., a high-quality solution) is determined from among the solutions found so far. Here, the elite solution is developed by applying the uniform crossover procedure to the first- and secondbest solutions obtained thus far. Iteration restarts with this newly developed elite solution. A fixed number of these processes are iteratively undertaken in TS-MSM.

(iii) Tabu Search with Multistart Method and LongTerm Memory (TS-MSM\&LM)

Intensification strategies are attained by means of long-term memory for exploiting features historically found desirable (e.g., Diaz and Fernadez 2001). In this study, a long-term memory scheme is performed using the following process. Ten elite solutions are extracted from the first- to the tenth-best solutions found thus far after a given number of iterations. The value of each gene is examined if it is identical to
Table 4 Computational Results for GLS, TS-Based Procedures, and RSM (20 Runs)

\begin{tabular}{lccccc}
\hline & GLS & TS-B & TS-MSM & TS-MSM\&LM & RSM \\
\hline Best & 1.45 & 1.45 & 1.45 & 1.45 & 1.02 \\
Average & 1.45 & 0.42 & 0.82 & 1.45 & 0.44 \\
Worst & 1.45 & 0.18 & 0.16 & 1.45 & 0.20 \\
Ave. CPU time (sec) & 481 & 1,230 & 1,209 & 560 & 1,271 \\
\hline
\end{tabular}

the ten elite solutions, and it is memorised and fixed if more than nine elite solutions commonly have the same value to restrict the move procedure to removing such common properties hereafter.

This type of strategy can be called the intermediateterm strategy to separate strictly from the longer-term diversification strategy (e.g., Dell'Amico, Lodi, and Maffioli 1999).

Parameter values for the TS operators are determined such that the maximum possible number of solutions to be searched is the same as that of GLS (i.e., $50 \times 30 \times 3=4,500$ solutions). The sizes of the tabu list are set to 8,5 , and 3 for TS-B, TS-MSM, and TS-MSM\&LM, respectively. The number of neighbourhoods is assumed to be 3 for TS-B, 7 for TS-MSM, and 5 for TS-MSM\&LM. Starting times are set to 5 for both TS-MSM and TS-MSM\&LM. TS-MSM\&LM also assumes a size of 30 for its long-term memory. These values were determined through a preliminary testing of parameter value settings.

The BCR values for the best, average, and worst solutions and the computational times required for GLS, TS-based procedures, and RSM are indicated in Table 4 . These results are obtained using different seeds of random values after 20 runs of each procedure. Average computational times were measured using a PC of 3.4 GHz CPU and 1.0 GB RAM.

It can be seen from the overall results shown in Table 4 that all three TS-based procedures offer better results than RSM. Results of the average solutions imply that both the multistart method and long-term memory scheme have better performance than the basic TS procedure. TS-MSM\&LM provides comparatively good results to GLS in terms of the best, average, and worst solutions found.

However, the average computational time in TSMSM\&LM is slightly higher than that of GLS. This difference could be significant when applied to a larger-sized transport network. Therefore, it can be concluded that GLS offers the better solution when applied to an actual large-size transport network even in a smaller search space of only 16 actions.

\section{Application Results}

The model is then applied to a large-scale transport network in the Philippines to investigate a possible development strategy for improving the interregional 


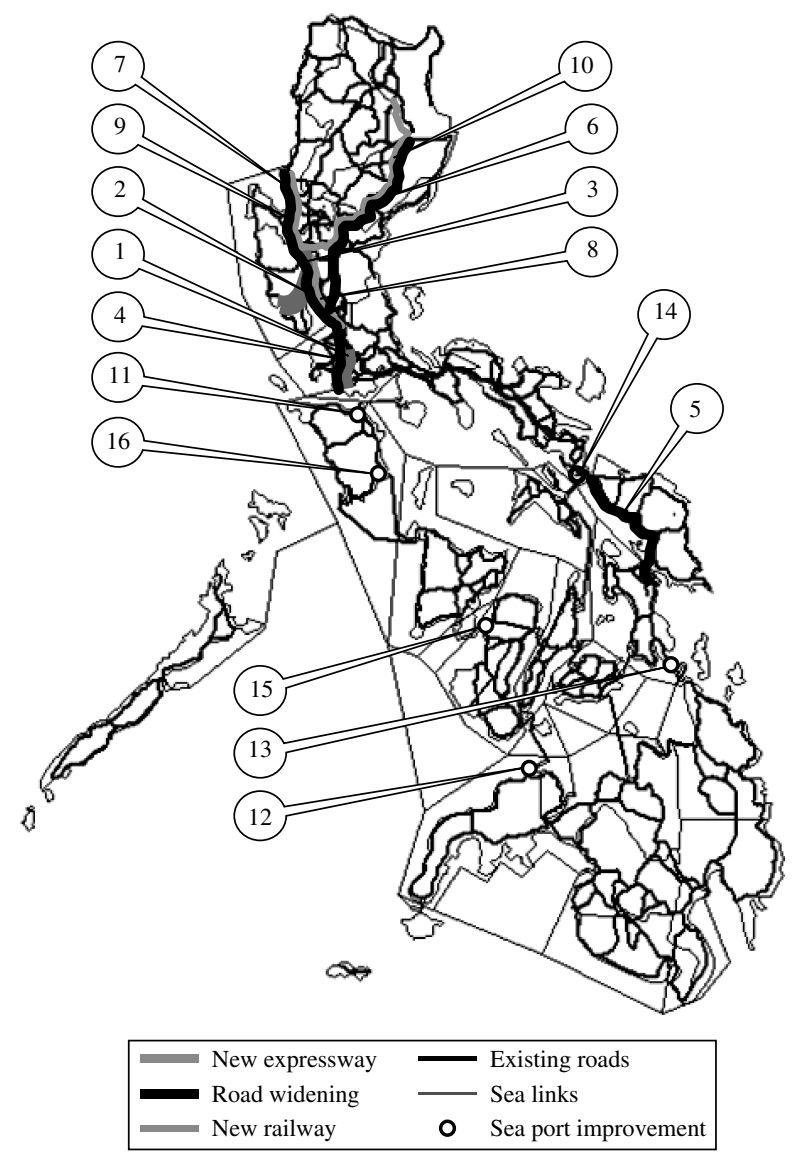

Figure 5 Modelled Network and Actions in the Philippines

freight transport network. The modelled transport network as illustrated in Figure 5 is composed of 424 nodes with 331 OD pairs for freight and 340 OD pairs for passenger, and 1,871 links comprising the national, provincial, and toll roads, railways, and portto-port sea routes. Inputs for the interregional freight and passenger movement consist of the data acquired from "The Inter-Regional Passenger and Freight Flow Surveys in the Republic of the Philippines (SIRPAFF)" study in 2004 conducted by the Department of Transportation and Communications (DOTC) and the Japan International Cooperation Agency (JICA).

Road capacities and speed data are obtained from the database of the Department of Public Works and Highways (DPWH). Data on fares incurred in roadbased freight transport is collected from private companies operating in the Philippines. Railway and sea network data and related information are acquired from the DOTC. Other characteristics of transport network is set in the same way as in the test network in West Java.

Sixteen multimodal transport projects are considered, including the development of new roads, railways, and upgrading or improvement of existing infrastructure. The projects are mainly situated along the major multimodal corridors in the Philippines (Figure 5).

\subsection{Flow Validation}

Prior to full computation, the modal split-assignment model in the lower level is first validated to confirm the flows resulting from the model and actual flow data and to make adjustments to model parameters. The performance of this type of multimodal multiclass user equilibrium assignment techniques has hardly ever been investigated with actual transport network and data, except for the work of Russ et al. (2005) attempt for the transport network in the entire Java Island, Indonesia.

Figure 6 compares the freight flow on each link estimated by the modal split-assignment model with that of what was actually observed in the national traffic flow and OD survey. The results shown in Figure 6 demonstrate that the estimated values from the model have a strong correlation with actual available data as indicated by correlation coefficients of more than 0.60 . Hence, the model is considered to perform well and can be used for predicting the major freight flows in accordance with available data, even though it is not a perfect fit and the number of data on rail freight flows may not be sufficient.

\subsection{Best Set of Actions}

The applicability of the model is tested using the transport network and actions in the Philippines. Figure 5 and Table 5 display 16 alternative planned actions including the establishment of new expressways and railways, road widening, and improvement of seaports. As can be seen in Figure 5, many of the actions are located in the major island of Luzon where Manila, the capital of the Philippines, is located close to action number 8. In most cases it is possible to choose the investment option that has positive BCR value, because each of the actions has positive benefit in this case study, even though the improvement of transport network might sometimes degrade its performance like Braess's paradox (Braess, Nagurney, and Wakolbinger 2005) and lead to the reduction in benefit.

A combination of four actions is found to be the best solution with a BCR value of 15.1. It includes action numbers 4 and 6 for road widening, and 15 and 16 for seaport improvement (i.e., action set $(4,6$, $15,16)$ ). This scheme could lead to less congested conditions for road-based freight transport, with a forecasted impact of $8.68 \%$ reduction in ton-kilometres for the road mode. This could also lead to a reduction in environmental impacts and energy consumption.

The result obtained from the model is plausible, because a cursory investigation of the existing conditions in the Philippines will reveal that most of 

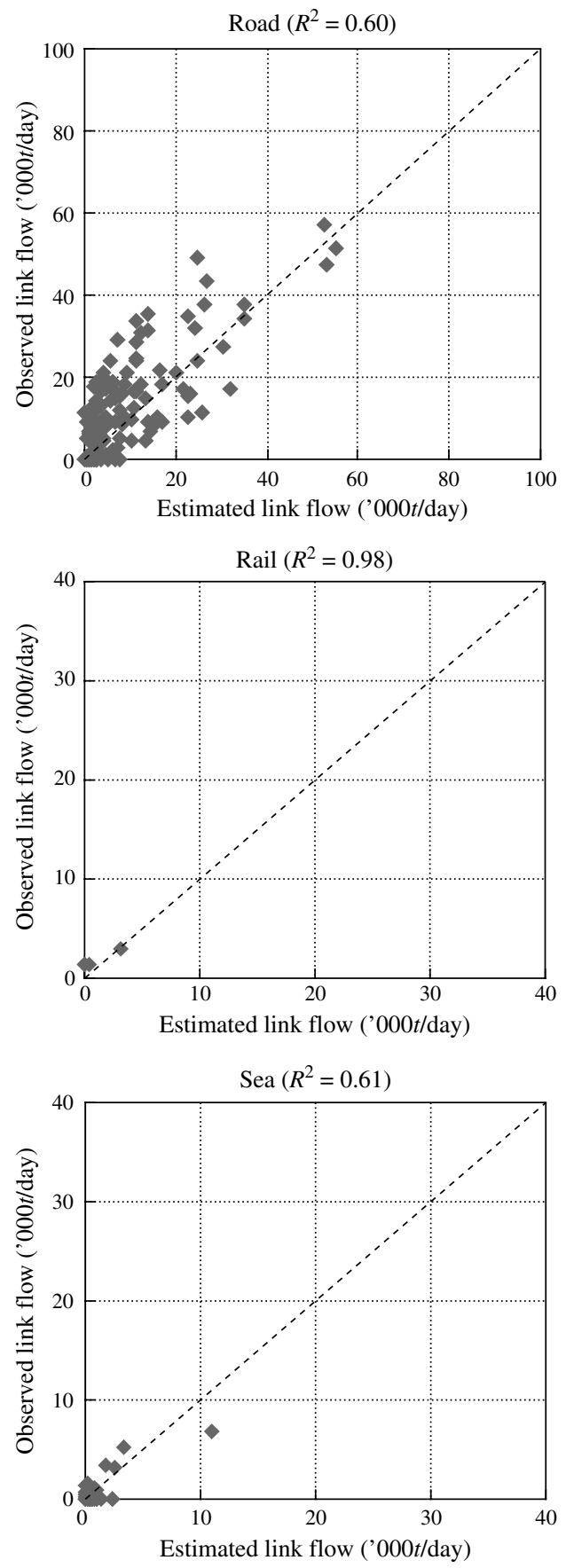

Figure $6 \quad$ Link Flow Validation Results

the road links are heavily loaded around the area where actions 4 and 6 are located. There is, therefore, increasing attention on the construction and rehabilitation of expressway connections around Manila. The additional capacity of these road links would obviously improve the traffic conditions in the area, although such projects would still require several years of planning, implementation, and construction. It is also fairly sensible that the best set of actions found contains seaport improvements (i.e., actions 15 and 16), because of the archipelagic nature of the Philippines. These actions would help establish the corridor running the length of the Philippines, which is currently called the Nautical Highway.

Figure 7 compares the BCR value, the total investment/operation cost (i.e., value in the denominator in Equation (5)), and the total freight-related benefit (i.e., value in the numerator in Equation (5)) for the best solution (i.e., actions 4, 6, 15, and 16), as well as other sets of actions around it. Either of the roadwidening actions of 4 and 6 or seaport improvement actions of 15 and 16 could provide significant benefits for freight transport. However, combining both types of actions apparently brings about synergistic effects as manifested by a considerably higher amount of benefits and BCR values, surpassing an increase in investment cost.

Additional road-related actions around Manila would not necessarily be useful in terms of BCR, even though these result in significant increases in freightrelated benefit. As seen in Figure 7, action set $(1,4,6$, $15,16)$ offers higher benefits than action set $(4,6$, $15,16)$ with a $10.3 \%$ reduction in ton-kilometres for the road mode. However, the investment cost required for establishing action 1 (new expressway) is relatively higher than those incurred for road widening and seaport improvement. Consequently, in terms of BCR, action set $(1,4,6,15,16)$ is less favorable than action set $(4,6,15,16)$.

The establishment of new railways could also have the potential to improve traffic conditions, especially with the increasing mode share for rail transport. However, rail-related actions are not selected in the best set of actions in this case. This is largely because of the significantly larger investment required for new railway establishment. This is proven by the results of action set $(4,6,8,15,16)$ displayed in Figure 7 . Additional investment cost is significantly required in adding action 8 (new railway) to the best action set of $(4,6,15,16)$, and therefore, action set $(4,6,8,15,16)$ results in lower BCR value than the best solution, even though it produces higher benefits with a $39.4 \%$ increase in ton-kilometres for the rail mode, as well as a $13.9 \%$ decrease for the road mode.

Figure 8 demonstrates the BCR values, total investment costs, and total freight-related benefits as a function of $\beta$, which is a factor relating to the amount of current freight movement in each OD pair (i.e., in case that the amount of freight movement in each OD pair is $\beta$ times as much as the amount of current one). Here, $\beta$ is assumed to range from 0.8 to 1.2. In Figure 8 , the action set on the left side for each value of $\beta$ represents the best solution found, and for comparison, the right side for each value illustrates the results for action set of $(4,6,15,16)$. 
Table 5 Listing of Actions (Case of Entire Philippines)

\begin{tabular}{rrcrr}
\hline & & & Investment/operation & \\
No. & Type of action & Location & $58,116.9$ & 0.55 \\
\hline 1 & New expressway & Calamba-Batangas & $124,267.0$ & 0.28 \\
2 & New expressway & Subic-San Fernando (Pampanga) & $100,496.0$ & 0.32 \\
3 & New expressway & Tarlac-Subic & $9,796.6$ & 3.68 \\
4 & Road widening & Manila-Batangas & $21,916.5$ & 1.61 \\
5 & Road widening & Allen-Tacloban & $31,466.9$ & 1.10 \\
6 & Road widening & Ilagan-Malolos & $24,067.7$ & 1.61 \\
7 & Road widening & San Fernando (La Union) -Caloocan & $398,451.9$ & 0.06 \\
8 & New railway & Manila-Angeles & 0.04 \\
9 & New railway & Angeles-San Fernando (La Union) & $659,242.0$ & 0.02 \\
10 & New railway & Tarlac-Tuguegarao & $1,234,191.5$ & 1.23 \\
11 & Seaport Improvement & Calapan & $10,548.8$ & 1.61 \\
12 & Seaport Improvement & Dapitan & $10,548.8$ & 1.31 \\
13 & Seaport Improvement & Liloan & $10,548.8$ & 1.28 \\
14 & Seaport Improvement & Matnog & $10,548.8$ & 1.59 \\
15 & Seaport Improvement & Pulupandan & $10,548.8$ & 1.32 \\
16 & Seaport Improvement & Roxas & $10,548.8$ & \\
\hline
\end{tabular}

*BCR of each action represents the value in case it is individually implemented.

The benefit gained from action set $(4,6,15,16)$ increases as the value of $\beta$ increases because of increased traffic congestion on the network. This action set reduces the road mode share by about $10 \%$ in each case. However, the best set of actions is not necessarily $(4,6,15,16)$ in terms of $\mathrm{BCR}$, except for the case of $\beta=1.0$, because the required investment cost has a significant influence on the BCR value. In the case of $\beta=0.8$, action set $(6,15)$ leads to almost the same amount of benefit as in action set $(4,6,15,16)$ with lower investment costs. This could largely be attributed to less congested conditions on the network caused by the reduction in OD freight volume. It would therefore be crucial not to oversupply infrastructure in less congested conditions.

In the case of $\beta=1.2$, action set $(12,15)$ also indicates higher BCR value with fewer actions even though the benefit obtained from it is significantly lower than action set $(4,6,15,16)$. Thus, investment cost greatly influences the BCR value, even though the costs required for seaport improvement are relatively lower. The higher BCR value could also be explained by more-congested conditions on the network because of increased OD freight volume that makes action set $(12,15)$ more efficient. Additional capacity in these seaport improvement actions could considerably mitigate traffic congestion arising within and around the seaports where increased freight movement exceeds their original capacity.

The best set of actions differs between $\beta=0.9$ and $\beta=1.1$ as well. These sets commonly include two road-widening actions in the central part of Luzon Island and two seaport improvement actions along the transport corridors of the Philippines. These actions can decrease total freight movement in terms of tonkilometres resulting from the reduction of road-based freight transport. Overall, it can be summarised that road widening in the central part of Luzon as well as seaport improvement along the Nautical Highway could be efficient in terms of freight-related BCR.

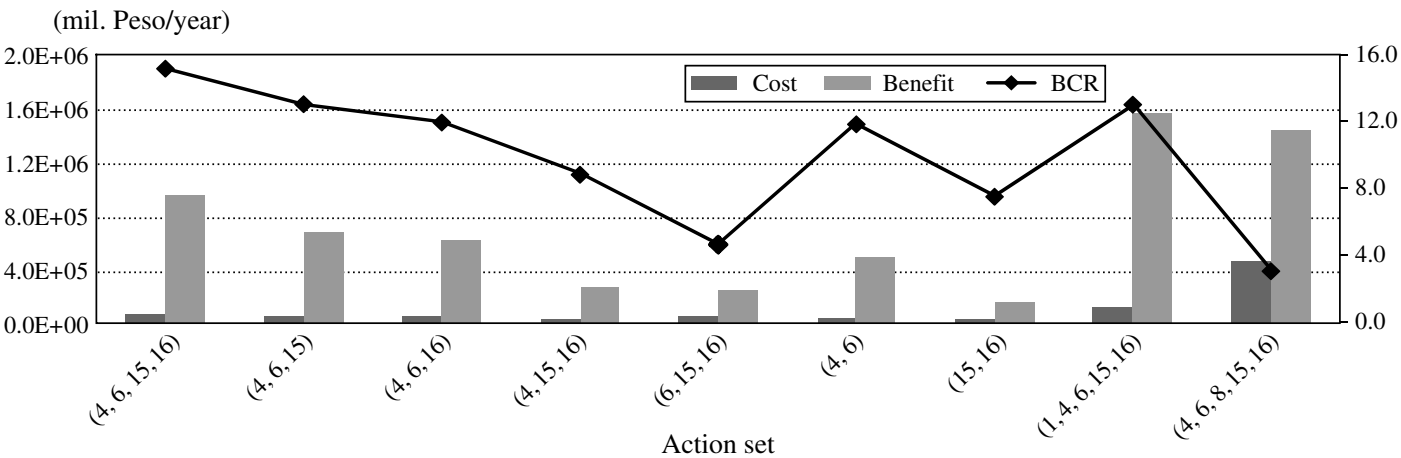

Figure 7 Computational Results Comparison Among Action Sets 


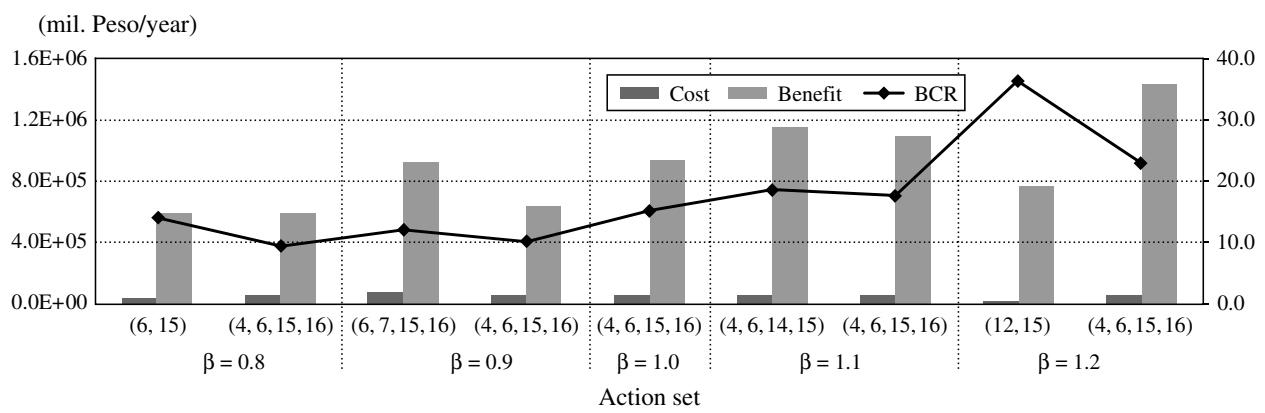

Figure 8 Computational Results Comparison Among Best Solutions Found

Note. $\beta$ : Factor for varying the amount of current freight movement in each OD pair.

\section{Conclusions}

This paper presented a model that can help efficiently expand the multimodal freight transport network system. It incorporates GLS, which is an evolutionary computation technique based on GA, for solving a discrete network design problem. The best combination of freight network improvement actions can be selected from among a feasible set of actions that includes improvement of existing infrastructure and establishment of new roads, rail, and sea links.

Modelling was undertaken within the framework of the bilevel programming problem, where the upper-level problem approximately optimises the combination of actions with metaheuristics-based procedures such that the freight-related benefit-cost ratio is maximised. Preliminary testing results with the transport network in West Java, Indonesia revealed that when the size of the transport network is relatively large and the search space is relatively small, GLS can offer the best performance among the GAbased and TS-based procedures tested, considering its robustness and faster searching ability.

The model was then applied to the transport network in the Philippines, where the development of a multimodal freight transport network is highly desired. The performance of the modal splitassignment model incorporated within the lower level was examined, and the model adequately represented the major freight flows based on available data. Results also indicated that improvement actions relating to road widening at heavy-loaded links and seaport improvement could be more effective than the development of new roads and rail links. This result could be significant in the development approach of multimodal freight transport network planning in archipelagic countries. Road widening in heavily congested areas, as well as seaport improvements, could be more effective in establishing the transport corridors running along and across the country.

These findings prove that the model proposed in this paper could provide adequate results in terms of the effective design of multimodal freight transport networks.

\section{References}

Aarts, E. H. L., J. H. M. Korst. 1989. Simulated Annealing and Boltzmann Machines: A Stochastic Approach to Combinatorial Optimization and Neural Computing. John Wiley \& Sons, Chichester, UK.

Ackley, D. H. 1987. A Connectionist Machine for Genetic Hillclimbing. Kluwer Academic Publishers, Boston.

Arnold, P., D. Peeters, I. Thomas. 2004. Modelling a rail/road intermodal transportation system. Transportation Res. Part E $\mathbf{4 0}$ 255-270.

Arroyo, J. E. C., V. A. Armentano. 2005. Genetic local search for multi-objective flowshop scheduling problems. Eur. J. Oper. Res. 151 717-738.

Aykin, T. 1990. On a quadratic integer program for the location of interacting hub facilities. Eur. J. Oper. Res. 46 409-411.

Balakrishnan, A., T. L. Magnanti, R. T. Wong. 1989. A dual-ascent procedure for large-scale uncapacitated network design. Oper. Res. 37 716-740.

Bard, J. F. 1998. Practical Bilevel Optimization: Algorithms and Applications. Kluwer Academic Publishers, Dordrecht, The Netherlands.

Boyce, D. E. 1984. Urban transportation network equilibrium and design models: Recent achievements and future prospectives. Environ. Planning 16 A 1445-1474.

Braess, D., A. Nagurney, T. Wakolbinger. 2005. On a paradox of traffic planning. Transportation Sci. 39 446-450.

Bruynooghe, M. 1972. An optimal method of choice of investments in a transport network. Presentation, Planning $\mathcal{E}$ Transport Research \& Computation Seminars on Urban Traffic Model Reasearch, London, UK.

Campbell, J. 1994. Integer programming formulations of discrete hub location problems. Eur. J. Oper. Res. 72 387-405.

Campbell, J. 1996. Hub location and the $p$-hub median problem. Oper. Res. 44 923-935.

Cascetta, E. 2001. Transportation Systems Engineering: Theory and Methods. Kluwer Academic Publishers, Dordrecht, The Netherlands.

Chen, M., A. S. Alfa. 1991. A network design algorithm using a stochastic incremental traffic assignment approach. Transportation Sci. 25 215-224.

Crainic, T. G., M. Florian, J. Leal. 1990. A model for the strategic planning of national freight transportation by rail. Transportation Sci. 24 1-24.

Dafermos, S. C. 1980. Traffic equilibrium and variational inequality. Transportation Sci. 14 43-54.

Daskin, M. S. 1995. Network and Discrete Location: Models, Algorithms, and Applications. John Wiley \& Sons, New York.

Davidor, Y. 1991. Genetic Algorithms and Robotics: A Heuristic Strategy for Optimization. World Scientific Publishing, Singapore.

Davis, L. 1991. Handbook of Genetic Algorithms. Van Nostrand, New York. 
Dell'Amico, M., A. Lodi, F. Maffioli. 1999. Solution of the cumulative assignment problem with a well-structured tabu search method. J. Heuristics 5 123-143.

Diaz, J. A., E. Fernadez. 2001. A tabu search heuristic for the generalized assignment problem. Eur. J. Oper. Res. 132 22-38.

Dorigo, M., T. Stutzle. 2004. Ant Colony Optimization. MIT Press, Boston.

Dorigo, M., G. Di Caro, L. M. Gambardella. 1999. Ant algorithms for discrete optimization. Artificial Life 5 137-172.

Drezner, Z. 1995. Facility Location: A Survey of Applications and Methods. Springer-Verlag, Heidelberg.

Florian, M., H. Spiess. 1982. The convergence of diagonalization algorithms for asymmetric network equilibrium problems. Transportation Res. Part B 16 477-483.

Francis, R. L., L. F. McGinnis, J. A. White. 1992. Facility Layout and Location: An Analytical Approach. Prentice-Hall, Upper Saddle River, NJ.

Friesz, T. L., R. L. Tobin, P. T. Harker. 1983. Predictive intercity freight network models: The state of the art. Transportation Res. Part A 17 409-417.

Galinier, P., J. K. Hao. 1999. Hybrid evolutionary algorithms for graph coloring. Combin. Optim. 3 379-397.

Gao, Z., J. Wu, H. Sun. 2005. Solution algorithm for the bi-level discrete network design problem. Transportation Res. Part B 39 479-495.

Glover, F., G. A. Kochenberger. 2003. Handbook of Metaheuristics. Kluwer Academic Publishers, Boston.

Glover, F., M. Laguna. 1997. Tabu Search. Kluwer Academic Publishers, Boston.

Glover, F., C. McMillan. 1986. The general employee scheduling problem: An integration of management science and artificial intelligence. Comput. Oper. Res. 15 563-593.

Goldberg, D. E. 1989. Genetic Algorithms in Search, Optimization, and Machine Learning. Addison Wesley, Reading, MA.

Guelat, J., M. Florian, T. G. Crainic. 1990. A multimode multiproduct network assignment model for strategic planning of freight flows. Transportation Sci. 24 25-39.

Herz, A., M. Widmer. 2003. Guidelines for the use of metaheuristics in combinatorial optimization. Eur. J. Oper. Res. 151 247-252.

Holland, J. H. 1975. Adaptation in Natural and Artificial Systems. University of Michigan Press, Ann Arbor, MI.

Jaszkiewicz, A. 2002. Genetic local search for multi-objective combinatorial optimization. Eur. J. Oper. Res. 137 50-71.

Jaszkiewicz, A., P. Kominek. 2003. Genetic local search with distance preserving recombination operator for a vehicle routing problem. Eur. J. Oper. Res. 151 352-364.

Kirkpatrick, S. C., D. Gellat, Jr., M. P. Vecchi. 1983. Optimization by simulated annealing. Science 220 671-680.

Laporte, G. 1988. Location-routing Problems. B. L. Golden, A. A. Assad, eds. Vehicle Routing: Methods and Studies. NorthHolland, Amsterdam, 163-198.

Luo, J. S., Z. Q. Pang, D. Ralph. 1996. Mathematical Programs with Equilibrium Constraints. Cambridge University Press, Cambridge, UK.

Magnanti, T. L., R. T. Wong. 1984. Network design and transportation planning: Models and algorithms. Transportation Sci. 18 $1-55$.

Melkote, S., M. S. Daskin. 2001. An integrated model of facility location and transportation network design. Transportation Res. Part A 35 515-538.

Merz, P., B. Freisleben. 1997. Genetic local search for the TSP: New results. Proc. 1997 IEEE Internat. Conf. Evolutionary Comput. IEEE Press, New York, 159-164.
Michalewicz, Z., D. B. Fogel. 2002. How to Solve It: Modern Heuristics. Springer-Verlag, Berlin.

Min, H., V. Jayaraman, R. Srivastava. 1998. Combined locationrouting problems: A synthesis and future research directions. Eur. J. Oper. Res. 108 1-15.

Murata, T., H. Ishibuchi. 1994. Performance evaluation of genetic algorithms for flowshop scheduling problems. Proc. 1st IEEE Internat. Conf. Evolutionary Comput. IEEE, Orlando, FL, 812-817.

Nagurney, A. 2000. Sustainable Transportation Networks. Edward Elgar, Northampton, UK

Nagurney, A., J. Dong. 2001. A multiclass, multicriteria traffic network equilibrium model with elastic demand. Transportation Res. Part B 36 445-469.

O'Kelly, M. 1987. A quadratic integer program for the location of interacting hub facilities. Eur. J. Oper. Res. 32 393-404.

Poorzahedy, H., M. A. Turnquist. 1982. Approximate algorithms for the discrete network design problem. Transportation Res. Part 16 45-55.

Priemus, H. 1999. On modes, nodes and networks: Technological and spatial conditions for a breakthrough towards multimodal terminals and networks of freight transport in Europe. Transportation Planning Tech. 23 83-103.

Radcliffe, N. J., P. D. Surry. 1994. Formal memetic algorithms. T. Fogarty, ed. Evolutionary Computing. Springer-Verlag, Berlin.

Ravi, R., A. Sinha. 2006. Approximation algorithms for problems combining facility location and network design. Oper. Res. 54 73-81.

Reeves, C. R. 1997. Genetic algorithms for the operations researcher. INFORMS J. Comput. 9 231-250.

Resende, M. G. C., J. Pinho de Sousa. 2004. Metaheuristics: Computer Decision-Making. Kluwer Academic Publishers, Dordrecht, The Netherlands.

ReVelle, C. S., G. Laporte. 1996. The plant location problem: New models and research prospects. Oper. Res. 44 864-874.

Ribeiro, C., P. Hansen. 2001. Essays and Surveys on Metaheuristics. Kluwer Academic Publishers, Dordrecht, The Netherlands.

Rochat, Y., E. D. Taillard. 1995. Probabilistic diversification and intensification in local search for vehicle routing. J. Heuristics 1 147-167.

Rothengatter, W. 1991. Cost-benefit-analyses for goods transport on roads. M. Kroon, R. Smit, J. Van Ham, eds. Freight Transport and the Environment. Elsevier, Amsterdam, 187-213.

Russ, B. F., T. Yamada, J. Castro, H. Yasukawa. 2005. Optimising the design of multimodal freight transport network in Indonesia. J. Eastern Asia Soc. Transportation Stud. 6 2894-2907.

Sheffi, Y. 1985. Urban Transportation Networks: Equilibrium Analysis with Mathematical Programming Methods. Prentice Hall, Englewood Cliffs, NJ.

Shepherd, S. P., A. Sumalee. 2004. A genetic algorithm based approach to optimal toll level and location problem. Networks Spatial Econom. 4(2) 161-179.

Southworth, F., B. E. Peterson. 2000. Intermodal and international freight modeling. Transportation Res. Part C 8 147-166.

Stackelberg, H. V. 1934. Marketform and Gleichgewicht. Julius Springer, Vienna.

Steenbrink, A. 1974. Transport network optimization in the Dutch integral transportation study. Transportation Res. Part B 8 11-27.

Syswerda, G. 1989. Uniform crossover in genetic algorithms. Proc. Third Internat. Conf. Genetic Algorithms, Morgan Kaufmann Publishers, Inc., Fairfax, VA, 2-9.

Taniguchi, E., M. Noritake, T. Yamada, T. Izumitani. 1999. Optimal size and location planning of public logistics terminals. Transportation Res. Part E 35 207-222. 
Taniguchi, E., R. G. Thompson, T. Yamada, J. H. R. Van Duin. 2001. City Logistics: Network Modelling and Intelligent Transport Systems. Pergamon, Oxford, UK.

Tavasszy, L. A. 1996. Modeling European freight transport flows. Unpublished doctoral dissertation, Delft University of Technology, Delft, The Netherlands.

Thomas, R. 1991. Traffic assignment techniques. Avebury Technical.

Yagiura, M., T. Ibaraki. 2001. On metaheuristic algorithms for combinatorial optimization problems. Systems Comput. Japan 32 33-55.
Yamada, T., E. Taniguchi, M. Noritake. 1999. Optimal location planning of logistics terminals based on multiobjective programming method. L. J. Sucharov, ed. Urban Transport V. WIT Press, Southampton, UK, 449-458.

Yang, H., M. G. H. Bell. 1998. Models and algorithms for road network design: A review and some new developments. Transport Rev. 18 257-278.

Zhang, X., H. Yang. 2004. The optimal cordon-based network congestion pricing problem. Transportation Res. Part B 38 517-537. 